\title{
Structural basis of recognition of SARS-CoV-2 by neutralizing antibodies isolated from convalescent patients
}

\author{
I Wilson ${ }^{1}, \mathrm{M} \mathrm{Yuan}^{2}, \mathrm{~N} \mathrm{Wu}{ }^{2}, \mathrm{H} \mathrm{Liu}^{2}, \mathrm{C} \mathrm{Lee}^{2}, \mathrm{X} \mathrm{Zhu^{2 }}$ \\ ${ }^{1}$ Dept of Integrative Structural \& Computational Biology, The Scripps ResearchInst, La Jolla, CA, \\ ${ }^{2}$ The Scripps Research Institute, La Jolla, CA \\ wilson@scripps.edu
}

SARS-CoV-2 has emerged as a global pandemic in 2020 with devastating health and socioeconomic consequences. Effective vaccines and therapeutics are urgently needed to combat this novel coronavirus and protect not only against this pandemic, but also potentially against related coronaviruses that may arise in the future. Many human neutralizing monoclonal antibodies (nAbs) have now been isolated from COVID-19 convalescent patients by research groups worldwide. Many of these nAbs target the receptor binding domain (RBD) of the spike protein, which engages the ACE2 receptor on human cells for viral entry. We have been determining crystal structures of nAbs bound to the spike protein RBD to determine their binding sites (epitopes) and possible mechanisms of neutralization. The nAb-RBD structural information can reveal the key sites of vulnerability on the virus. Recurring motifs as well as novel binding modes used by antibodies for recognition of the RBD have been delineated, including which antibody germline genes in the human immune repertoire are preferentially used to target the RBD. Such structural and functional information can be utilized to assess vaccine responses against SARS CoV-2, aid in modifications and improvement of vaccine immunogens, and for design of novel therapeutics that inhibit entry of SARS CoV-2 into host cells.

Acta Cryst. (2020). A76, a202 